\title{
Preclinical Evaluation of Genexol-PM, a Nanoparticle Formulation of Paclitaxel, as a Novel Radiosensitizer for the Treatment of Non-Small Cell Lung Cancer
}

\author{
Michael E. Werner, $\mathrm{PhD}^{\star}, \dagger$, Natalie D. Cummings, $\mathrm{BS}^{*}, \dagger$, Manish Sethi, $\mathrm{PhD}^{\star}, \dagger$, Edina C. \\ Wang, BS ${ }^{\star}, \dagger$, Rohit Sukumar, BS ${ }^{*}, \dagger$, Dominic T. Moore, MPH ${ }^{\ddagger}$, and Andrew Z. Wang, MD ${ }^{*}, \ddagger$ \\ *Laboratory of Nano- and Translational Medicine, Department of Radiation Oncology, Lineberger \\ Comprehensive Cancer Center, University of North Carolina-Chapel Hill, Chapel Hill, North \\ Carolina \\ †Carolina Center for Cancer Nanotechnology Excellence, University of North Carolina-Chapel Hill, \\ Chapel Hill, North Carolina \\ ‡Division of Biostatistics and Data Management, Lineberger Comprehensive Cancer Center, \\ University of North Carolina-Chapel Hill, Chapel Hill, North Carolina
}

\begin{abstract}
Purpose-A key research objective in radiation oncology is to identify agents that can improve chemoradiation therapy. Nanoparticle (NP) chemotherapeutics possess several properties, such as preferential accumulation in tumors, that are uniquely suited for chemoradiation therapy. To facilitate the clinical translation of NP chemotherapeutics in chemoradiation therapy, we conducted preclinical evaluation of Genexol-PM, the only clinically approved NP chemotherapeutic with a controlled drug release profile, as a radiosensitizer using non-small cell lung cancer (NSCLC) as a model disease.
\end{abstract}

Methods and Materials-The physical characteristics and drug release profile of Genexol-PM were characterized. Genexol-PM's efficacy as a radiosensitizer was evaluated in vitro using NSCLC cell lines and in vivo using mouse xenograft models of NSCLC. Paclitaxel dose to normal lung and liver after Genexol-PM administration were quantified and compared with that after Taxol administration.

Results-Genexol-PM has a size of $23.91 \pm 0.41 \mathrm{~nm}$ and surface charge of $-8.1 \pm 3.1 \mathrm{mV}$. It releases paclitaxel in a controlled release profile. In vitro evaluation of Genexol-PM as a radiosensitizer showed it is an effective radiosensitizer and is more effective than Taxol, its small molecule counterpart, at the half maximal inhibitory concentration. In vivo study of Genexol-PM as a radiosensitizer demonstrated that it is more effective as a radiosensitizer than Taxol. We also found that Genexol-PM leads to lower paclitaxel exposure to normal lung tissue than Taxol at 6 hours postad-ministration.

Conclusions-We have demonstrated that Genexol-PM is more effective than Taxol as a radiosensitizer in the preclinical setting and holds high potential for clinical translation. Our data support the clinical evaluation of Genexol-PM in chemoradiation therapy for NSCLC.

(C) 2013 Elsevier Inc. All rights reserved.

Reprint requests to: Andrew Z. Wang, MD, 101 Manning Dr, Campus Box 7512, Chapel Hill, NC 27599. Tel: (919) 445-5208; zawang@med.unc.edu.

Conflict of interest: A.Z.W. is a consultant for Samyang Biopharmaceuticals, Inc.

Supplementary material for this article can be found at www.redjournal.org. 


\section{Introduction}

The concurrent administration of chemotherapy and radiation therapy, also called chemoradiation therapy, is an important treatment paradigm in the curative management of many cancers (1). Chemoradiation therapy has not only consistently shown improved local tumor control but also improves survival when compared with either sequential treatment or sole administration of chemotherapy or radiation therapy in some malignancies $(1,2)$. However, chemoradiation therapy is not without limitations. The concurrent use of both chemotherapy and radiation therapy has significantly higher toxicities compared with either treatment alone or sequential use (3). Thus, the development of agents and approaches to further improve the therapeutic index of chemoradiation therapy has been a major research objective. A key challenge in this effort has been to selectively deliver chemotherapeutics to tumors while minimizing drug dose to normal tissue. Although traditional drug delivery techniques have failed to address this challenge (4), the development of nanoparticle (NP) formulations of chemotherapeutics offers an unprecedented opportunity. NP therapeutics possess important characteristics that are uniquely suited for chemoradiotherapy. NPs, as macromolecules, preferentially accumulate in tumors through the enhanced permeability and retention (EPR) effect, leading to high intratumoral drug concentrations (5). NPs are also unable to penetrate normal vasculatures and capillaries, thus leading to lower drug doses to normal tissues compared with their small molecule counterparts. Moreover, NP therapeutics have been found to have lower systemic toxicity than small molecules (6). Lastly, NP formulations of chemotherapeutics can release their cargo in a controlled fashion (5). Such prolonged release can increase the synergistic effects of chemotherapy and radiation therapy. Indeed, several preclinical studies have demonstrated that NP chemotherapeutics can improve the therapeutic efficacy of chemoradiotherapy $(7,8)$. However, there have been no reports evaluating NP therapeutics that are clinically approved or under-going clinical investigation for use in chemoradiation therapy. Such studies are necessary for clinical translation of NP therapeutics in chemoradiation therapy. To fill this knowledge gap, we conducted a preclinical evaluation of Genexol-PM, the only clinically approved secondgeneration NP chemotherapeutic with a controlled drug release profile, in chemoradiation therapy using non-small cell lung cancer (NSCLC) as a model disease.

Genexol-PM is a polymeric NP micelle formulation of paclitaxel that has been approved in South Korea for the treatment of breast cancer and $\operatorname{NSCLC}(9,10)$. It is composed of lowmolecular-weight amphiphilic diblock copolymer, monomethoxy poly (ethylene glycol)block-poly(D,L-lactide) (mPEG-PDLLA) and paclitaxel (11). Genexol-PM has shown lower toxicity than Taxol with its maximum tolerated dose identified as 2 to 3 times that of Taxol (9-11). Given that chemoradiation therapy is often used for locally advanced and unresectable NSCLC and paclitaxel has been shown be an excellent radiosensitizer in NSCLC, we chose to study Genexol-PM in chemoradiation therapy using NSCLC as a model disease. Moreover, chemoradiation therapy for NSCLC has significant toxicities with approximately $5 \%$ to $10 \%$ of patients dying from treatment-related pulmonary toxicities (3, 12). Thus, there is a strong clinical need to improve chemoradiation therapy for NSCLC. In this study, Genexol-PM was compared with Taxol in chemoradiation therapy in vitro using NSCLC cell lines and in vivo using mouse flank xenograft models of NSCLC. We also quantified the paclitaxel dose delivered to normal lung and liver tissues after Genexol-PM and Taxol administration.

\section{Methods and Materials}

\section{Materials}

Genexol-PM was provided by Samyang Biopharmaceuticals Corporation (Seoul, Korea) as a gift. Each gram of Genexol-PM contains $115 \mathrm{mg}$ of paclitaxel. Genexol-PM was 
resuspended in phosphate buffer saline based on clinical administration protocol before use. Taxol $6 \mathrm{mg} / \mathrm{mL}$ injectable solution (Teva, Sellersville, PA) was purchased from the University of North Carolina Hospital pharmacy.

\section{Characterization of Genexol-PM}

Genexol-PM size (diameter, $\mathrm{nm}$ ) and surface charge $(\xi$-potential, $\mathrm{mV})$ were characterized using a ZetaPALS dynamic light scattering detector (Brookhaven Instruments, Holtsville, NY). Transmission electron microscopy (TEM) images of Genexol-PM were obtained at the University of North Carolina (UNC) Microscopy Services Laboratory Core Facility. NPs were stained using a phosphotungstate stain before TEM imaging.

\section{Paclitaxel release characterization}

To measure the release profile of paclitaxel from Genexol-PM, $0.1 \mathrm{~mL}$ of Genexol-PM solution at a concentration of $1 \mathrm{mg} / \mathrm{mL}$ was aliquot equally into Slide-A-Lyzer MINI dialysis microtubes with a molecular weight cut-off of $10 \mathrm{kDa}$ (Pierce, Rockford, IL) and subjected to dialysis as described previously (7). Paclitaxel content was quantitatively analyzed using an Agilent 1100 HPLC (Paolo Alto, CA) equipped with a C18 chromolith flash column (Merck, Darmstadt, Germany). Paclitaxel absorbance was measured by a UVVIS detector at $227 \mathrm{~nm}$ and a retention time of $8.5 \mathrm{~min}$ in $0.25 \mathrm{~mL} / \mathrm{min}$ gradient (from 0:100 to $100: 0)$ of acetonitrile/water.

\section{Cell culture}

NSCLC cell lines were acquired from the Lineberger Comprehensive Cancer Center Tissue Culture Facility. A549 cells were cultured in Dulbecco's Modified Eagle Medium (DMEM) (Gibco, Invitrogen, Carlsbad, CA) supplemented with 10\% fetal bovine serum (Mediatech, Manassas, VA) and penicillin/streptomycin (Mediatech). H460 was cultured in RPMI 1640 (Gibco) with $10 \%$ fetal bovine serum (FBS), $2 \mathrm{mM} \mathrm{L}$-glutamine, $1.5 \mathrm{~g} / \mathrm{L} \mathrm{Na}$ bicarbonate, 10 mM HEPES (4-(2-hydroxyethyl)-1-piper-azineethanesulfonic acid) buffer, $1 \mathrm{mM} \mathrm{Na}$ pyruvate, penicillin/streptomycin (Mediatech), $4.5 \mathrm{~g} / \mathrm{L}$ glucose.

\section{In vitro x-ray irradiation}

Radiation therapy was given using a Precision X-RAD 320 (Precision X-Ray, North Branford, CT) operating at $320 \mathrm{kvp}$ and $12.5 \mathrm{~mA}$ as described previously (7).

\section{Clonogenic survival assay}

Cells were seeded at densities ranging from 100 to 200,000 cells in $4 \mathrm{~mL}$ of culture medium in 25-mL flasks 1 day before treatment. Cells were treated with $\mathrm{IC}_{50}$ doses of Taxol (1.77 $\mathrm{ug} / \mathrm{mL}$ for A549 and $17.7 \mathrm{ug} / \mathrm{mL}$ for H460) or Genexol-PM (15 ug/mL for A549 and 150 $\mathrm{ug} / \mathrm{mL}$ for H460) for 1 hour and washed 3 times with fresh media after incubation. Cells were irradiated with 2 fractions of $0,1,2$, or 3 Gy separated by 12 hours. Cells were incubated and counted as described previously (7). Data were analyzed using Origin Pro 8.6 software. Polynomial curve fitting was performed using second polynomial order.

\section{In vivo tumor assay}

Tumors were established in the upper dorsal region of $\mathrm{Nu} / \mathrm{Nu}$ mice by injecting $1 \times 10^{6}$ of H460 or A549 cells in a 1:1 RPMI:Matrigel solution. Tumors were incubated for 10 days (H460) or 14 days (A549) to reach approximate tumor volume of $100 \mathrm{~mm}^{3}$ before treatment. Mice (7 per experimental group) were treated intravenously with Taxol $(2.5 \mathrm{mg} / \mathrm{kg})$ or Genexol-PM $(21.5 \mathrm{mg} / \mathrm{kg})$ and subsequently irradiated 6 hours postinjection. The mice were randomized into experimental groups before treatment. The dose rate at a source-subject distance of $70 \mathrm{~cm}$ was $50 \mathrm{cGy} / \mathrm{min}$. Mice were irradiated with 5 daily fractions of $3 \mathrm{~Gy}$. The 
head and abdomen regions of mice were shielded using $0.5 \mathrm{~cm}$ of lead. Only the truncal region containing tumor xenograft was irradiated. Tumor volumes were calculated by measuring 2 perpendicular diameters with a caliper and using the formula of $\mathrm{V}=0.5 \times \mathrm{a} \times$ $b^{2}$ where, $a$ and $b$ are the larger and smaller diameters, respectively. The tumors were measured every 2 days, and the relative percent change in tumor volume was calculated using the relation $100 *\left(\mathrm{~V}_{\mathrm{i}}-\mathrm{V}_{\mathrm{o}}\right) / \mathrm{V}_{\mathrm{o}}$, where $\mathrm{V}_{\mathrm{i}}$ is the volume calculated and $\mathrm{V}_{\mathrm{o}}$ is the initial volume on day 1 .

Tumor volume was measured every other day until the tumor reached 3 times the initial volume or $2 \mathrm{~cm}$ in the maximum dimension, at which point the animal was euthanized. All animal work was approved and monitored by the UNC Animal Care and Use Committee.

\section{Statistical methods}

We used response features analysis to analyze our serial measures of tumor growth (13). This method allows for the comparison of individual growth delay profiles by using summary measures. The particular summary measure used was the AUC (or area under the curve), which was particularly appropriate for the growth-delay profiles we encountered. The Wilcoxon 2-group method (using Van der Waerden normal scores) was used to test whether there was a significant difference in AUCs between chemoradiation therapy with Genexol-PM compared with chemoradiation therapy with Taxol. Statistical analyses were performed using SAS statistical software, version 9.2, from the SAS Institute (Cary, NC).

\section{Quantification of paclitaxel dose in tissue}

Mice bearing H460 tumors ( 5 animals per group) were treated intravenously with equivalent paclitaxel dose of Taxol $(2.5 \mathrm{mg} / \mathrm{kg})$ or Genexol-PM $(21.5 \mathrm{mg} / \mathrm{kg})$. Mice were euthanized after $6 \mathrm{~h}$ or $24 \mathrm{~h}$, and organs were collected and weighed. Organs were homogenized 4:1 (v/ w) in chloroform to extract paclitaxel. Homogenate was centrifuged, and the organic layer was collected, evaporated, and reconstituted in $0.1 \%$ acetic acid in methanol for analysis via liquid chromatography-mass spectrometry using an Applied Biosystems API 4000 triple quadrupole mass spectrometer with an atmospheric pressure chemical ionization interface.

\section{Results}

\section{Characterization of Genexol-PM}

Genexol-PM's physical properties were characterized as these properties have not been described in previous publications. Genexol-PM has a size of $23.91 \pm 0.41 \mathrm{~nm}$ by dynamic light scattering. The NPs are also monodispersewith a polydispersity index of $0.08 \pm 0.02$. The NP size and dispersity were confirmed by TEM (Fig. 1A). We found that Genexol-PM has a surface charge of $-8.1 \pm 3.1 \mathrm{mV}$. We also studied the drug release profile of GenexolPM. Paclitaxel release from the Genexol-PM demonstrated first-order controlled release kinetics with $\sim 65 \%$ drug released at 24 hours and $95 \%$ drug release at 48 hours (Fig. 1B).

\section{In vitro radiosensitization with Genexol-PM}

The therapeutic efficacy of Genexol-PM as a radiosensitizer in vitro was evaluated in 2 commonly studied NSCLC cell lines H460 (large cell) and A549 (adenocarcinoma). To determine the sensitivity of each cell line to Genexol-PM, a dose-response curve was performed with no radiation therapy for each cell line (Fig. S1). Radio-sensitization experiments were conducted using $\mathrm{IC}_{50}$ concentrations as well as equivalent paclitaxel concentrations of Genexol-PM and Taxol. Clonogenic survival curves of cells after combined Genexol-PM and fractionated radiation therapy were generated for each cell line. They were compared to that of fractionated radiation therapy alone. We demonstrated that despite the lack of EPR effect in vitro, Genexol-PM is an effective radiosensitizer and is 
more effective than Taxol in both cell lines at $\mathrm{IC}_{50}$ doses (Fig. 2). The sensitizer enhancement ratio (SER) of Taxol at 10\% survival in H460 cells is 1.03, whereas the SER of Genexol-PM is 1.12. The SER is greater in A549 cells at 10\%; with Taxol at 1.12 and Genexol-PM at 1.23. Also, Genexol-PM was as effective a radiosensitizer as Taxol at equivalent paclitaxel doses (Fig. S2). These results confirmed that Genexol-PM is an effective radiosensitizer.

\section{In vivo radiosensitization with Genexol-PM}

To validate the in vitro result and to evaluate the efficacy of Genexol-PM as a radiosensitizer in vivo, we used a murine flank xenograft model of NSCLC. Mice bearing either H460 or A549 cell xenograft tumors were treated with Genexol-PM, Taxol, or saline followed by radiation therapy. After treatment with 5 daily fractions of $3 \mathrm{~Gy}$, the tumor growth delay curves demonstrated that chemoradiation therapy with Genexol-PM leads to significantly longer tumor growth delay compared with chemoradiotherapy with Taxol in H460 cells ( $P=.008$; Fig. 3A). Although the difference between the 2 treatments does not appear as significant in A549 cells $(P=.18$; Fig. 3B), the impression remains that chemoradiation therapy with Genexol-PM is more effective. Since tumors that were treated with chemotherapy only (Genexol-PM or Taxol) did not show significant difference in growth delay, the difference between Genexol-PM+XRT and Taxol+XRT is likely due to more effective radiosensitization by Genexol-PM (Fig. S3). Our results showed the high potential of Genexol-PM in improving chemoradiation therapy.

\section{Evaluation of normal tissue drug exposure}

Pulmonary toxicity is the main cause of mortality and morbidity from chemoradiation therapy for NSCLC. It is caused by normal lung tissue receiving both chemotherapy and radiation therapy. To determine whether Genexol-PM can lead to lower normal lung paclitaxel exposure, we compared the paclitaxel concentrations in mouse lungs after Genexol-PM and Taxol administration. We found that Genexol-PM leads to significantly lower lung paclitaxel concentration ( $P=.01$ ) acutely after injection (6 hours). This difference in drug concentration disappeared at 24 hours (Fig. 4). Given that NPs are generally cleared through hepatic clearance and the concern over hepatotoxicity, we also compared the paclitaxel concentration in mouse liver after Genexol-PM and Taxol administration. Although not statistically significant, Genexol-PM lead to lower paclitaxel exposure to mouse liver than paclitaxel at 6 hours $(P=30)$. Similar to the drug exposure in the lung, there was no difference in liver paclitaxel concentration between Genexol-PM and Taxol at 24 hours.

\section{Discussion}

Advances in nanomedicine have enabled the development of many NP-based chemotherapeutics (14). Although they have been widely used as chemotherapeutics, there has been limited clinical evaluation of these drugs in chemoradiation therapy because of toxicity concerns (15). Recent nanomedicine clinical translation efforts have focused on the development of NP formulation of taxanes, a class of chemotherapeutics that is also used in chemoradiation. Today, there are 2 clinically approved NP taxane formulations, nabpaclitaxel (Abraxane) and Genexol-PM, with several additional therapeutics under clinical investigation (paclitaxel poliglumex [Opaxio], BIND-014 [biologically targeted polymeric NP docetaxel]) (5). Among these, Genexol-PM is the only NP therapeutic that possesses controlled drug release and is approved for clinical use. Furthermore, the only other approved NP taxane, nab-paclitaxel (nanoparticle albumin-bound paclitaxel), dissociates upon administration and cannot take full advantage of the EPR effect (16). Therefore, 
Genexol-PM is the best NP therapeutic candidate for clinical translation in chemoradiation therapy.

Preclinical studies have shown that the optimal NP physical characteristics for tumor targeting are sizes $<100 \mathrm{~nm}$ with negative surface charges (17). Genexol-PM's properties $(23.0 \pm 4.5 \mathrm{~nm}$ and $-8.1 \pm 3.1 \mathrm{mV})$ suggest that it is excellent for tumor targeting. Another advantage of using Genexol-PM in chemoradiotherapy is its controlled drug release. Although previous clinical investigations have demonstrated that prolonged drug exposure can increase the therapeutic efficacy of chemoradiation therapy (18), most small molecule chemotherapeutics, including taxanes, are too toxic to be administered continuously. NP therapeutics, conversely, can increase drug exposure time in tumors without increasing toxicity, both to local healthy tissue and systemically. In this study, we found Genexol-PM releases paclitaxel in a controlled and slow fashion, with only $40 \%$ drug release after 16 hours and $>90 \%$ of drug release after 48 hours. Such slow drug release should lead to increased synergistic effects between paclitaxel and radiation therapy, which in turn should result in improved therapeutic efficacy.

The efficacy of Taxol as a radiosensitizer has been evaluated previously (19). Our study compared the efficacy of Genexol-PM as a radiosensitizer with that of Taxol. At the $\mathrm{IC}_{50}$ dose, Genexol-PM is more effective than Taxol, whereas at the same paclitaxel dose, Genexol-PM is as effective as Taxol in vitro. The in vitro results reflect the fact that the advantages of NP therapeutics in chemoradiation therapy are controlled drug release and biodistribution but only the effects of controlled drug release can be observed in the in vitro setting. Genexol-PM was also evaluated in vivo as a radiosensitizer in mouse xenograft models of NSCLC. We demonstrated that at equivalent doses of paclitaxel, Genexol-PM led to significantly longer tumor growth delay than Taxol in mice treated with chemoradiation therapy. It is also important to note that we used a relatively low dose of paclitaxel in this study. This is shown by the lack of tumor growth delay in mice treated with only Taxol or Genexol-PM compared with mice that received no treatment. Our findings confirm that Genexol-PM is an effective radiosensitizer. On the basis of NP biodistribution properties, we also hypothesized that Genexol-PM would lead to lower paclitaxel dose to lungs when compared with Taxol. Indeed, paclitaxel dose in mouse lungs was significantly lower in mice given Genexol-PM than that in mice given Taxol at 6 hours after chemotherapy administration. Because pulmonary toxicity from chemoradiation therapy is related to paclitaxel dose in normal lung tissue (20), such reduction in paclitaxel dose can result in lower pulmonary toxicity.

In summary, we have demonstrated that Genexol-PM holds high potential as a radiosensitizer in chemoradiation therapy for NSCLC. Our findings provide the preclinical evidence to support the clinical evaluation of Genexol-PM as part of the chemoradiation therapy regimen for NSCLC. Genexol-PM can be incorporated into existing chemoradiation therapy regimens in place of Taxol. Results from this study suggest that substituting Taxol with Genexol-PM can improve the therapeutic ratio of treatment. More broadly, our report and previously reported preclinical studies suggest that NP chemotherapeutics are more effective than their small-molecule counterparts as radio-sensitizers. Therefore, the clinical development of novel NP chemotherapeutics should include clinical evaluations in chemoradiation therapy. Lastly, given the importance of the chemoradiation therapy treatment paradigm, nanomedicine research efforts should focus on the development of novel NP therapeutics with the primary application of improving chemoradiation therapy.

\section{Supplementary Material}

Refer to Web version on PubMed Central for supplementary material. 


\section{Acknowledgments}

We thank the Microscopy Services Laboratory, Animal Studies Core, and the Analytical Chemistry Core (School of Pharmacy) at the University of North Carolina for their assistance with procedures. We also thank E. Claire Dees for advice on this manuscript.

This work was supported by the University Cancer Research Fund from the University of North Carolina, a North Carolina Translational and Clinical Sciences Institute (NC TraCS) pilot grant, and a Lung Cancer Research Foundation Grant. A.Z.W. was supported by National Institutes of Health/National Cancer Institute K12 Career Development Award 5-K12-CA120780-01-05 and National Institutes of Health Center for Nanotechnology Excellence Grant 1-U54-CA151652-01.

\section{References}

1. Seiwert TY, Salama JK, Vokes EE. The concurrent chemoradiation paradigm—general principles. Nat Clin Pract Oncol. 2007; 4:86-100. [PubMed: 17259930]

2. Eifel PJ. Chemoradiotherapy in the treatment of cervical cancer. Semin Radiat Oncol. 2006; 16:177-185. [PubMed: 16814159]

3. Forastiere AA, Goepfert H, Maor M, et al. Concurrent chemotherapy and radiotherapy for organ preservation in advanced laryngeal cancer. N Engl J Med. 2003; 349:2091-2098. [PubMed: 14645636]

4. Rasch CR, Hauptmann M, Schornagel J, et al. Intra-arterial versus intravenous chemoradiation for advanced head and neck cancer: Results of a randomized phase 3 trial. Cancer. 2010; 116:21592165. [PubMed: 20187094]

5. Wang AZ, Langer RS, Farokhzad OC. Nanoparticle Delivery of Cancer Drugs. Annu Rev Med. 2012; 63:185-198. [PubMed: 21888516]

6. O'Brien ME, Wigler N, Inbar M, et al. Reduced cardiotoxicity and comparable efficacy in a phase III trial of pegylated liposomal doxorubicin $\mathrm{HCl}$ (CAELYX/Doxil) versus conventional doxorubicin for first-line treatment of metastatic breast cancer. Ann Oncol. 2004; 15:440-449. [PubMed: 14998846]

7. Werner ME, Copp JA, Karve S, et al. Folate-targeted polymeric nanoparticle formulation of docetaxel is an effective molecularly targeted radiosensitizer with efficacy dependent on the timing of radiotherapy. ACS Nano. 2011; 5:8990-8998. [PubMed: 22011071]

8. Jung J, Park SJ, Chung HK, et al. Polymeric nanoparticles containing taxanes enhance chemoradiotherapeutic efficacy in nonesmall cell lung cancer. Int J Radiat Oncol Biol Phys. 2012; 84:e77-e83. [PubMed: 22795728]

9. Lee KS, Chung HC, Im SA, et al. Multicenter phase II trial of Genexol-PM, a Cremophor-free, polymeric micelle formulation of paclitaxel, in patients with metastatic breast cancer. Breast Cancer Res Treat. 2008; 108:241-250. [PubMed: 17476588]

10. Kim DW, Kim SY, Kim HK, et al. Multicenter phase II trial of Genexol-PM, a novel Cremophorfree, polymeric micelle formulation of paclitaxel, with cisplatin in patients with advanced nonsmall-cell lung cancer. Ann Oncol. 2007; 18:2009-2014. [PubMed: 17785767]

11. Lim WT, Tan EH, Toh CK, et al. Phase I pharmacokinetic study of a weekly liposomal paclitaxel formulation (Genexol-PM) in patients with solid tumors. Ann Oncol. 2010; 21:382-388. [PubMed: 19633055]

12. Fournel P, Robinet G, Thomas P, et al. Randomized phase III trial of sequential chemoradiotherapy compared with concurrent chemoradiotherapy in locally advanced non-small-cell lung cancer: Groupe Lyon-Saint-Etienne d'Oncologie Thoracique-Groupe Francais de Pneumo-Cancerologie NPC 95-01 Study. J Clin Oncol. 2005; 23:5910-5917. [PubMed: 16087956]

13. Matthews JN, Altman DG, Campbell MJ, et al. Analysis of serial measurements in medical research. BMJ. 1990; 300:230-235. [PubMed: 2106931]

14. Zhang L, Gu FX, Chan JM, et al. Nanoparticles in medicine: Therapeutic applications and developments. Clin Pharmacol Ther. 2008; 83:761-769. [PubMed: 17957183]

15. Burris HA 3rd, Hurtig J. Radiation recall with anticancer agents. Oncologist. 2010; 15:1227-1237. [PubMed: 21045191] 
16. Guarneri V, Dieci MV, Conte P. Enhancing intracellular taxane delivery: Current role and perspectives of nanoparticle albumin-bound paclitaxel in the treatment of advanced breast cancer. Expert Opin Pharmacother. 2012; 13:395-406. [PubMed: 22263900]

17. Kamaly N, Xiao Z, Valencia PM, et al. Targeted polymeric therapeutic nanoparticles: Design, development and clinical translation. Chem Soc Rev. 2012; 41:2971-3010. [PubMed: 22388185]

18. O'Connell MJ, Martenson JA, Wieand HS, et al. Improving adjuvant therapy for rectal cancer by combining protracted-infusion fluorouracil with radiation therapy after curative surgery. $\mathrm{N}$ Engl $\mathrm{J}$ Med. 1994; 331:502-507. [PubMed: 8041415]

19. Liebmann J, Cook JA, Fisher J, et al. In vitro studies of Taxol as a radiation sensitizer in human tumor cells. J Natl Cancer Inst. 1994; 86:441-446. [PubMed: 7907149]

20. Taghian AG, Assaad SI, Niemierko A, et al. Risk of pneumonitis in breast cancer patients treated with radiation therapy and combination chemotherapy with paclitaxel. J Natl Cancer Inst. 2001; 93:1806-1811. [PubMed: 11734597] 
A)

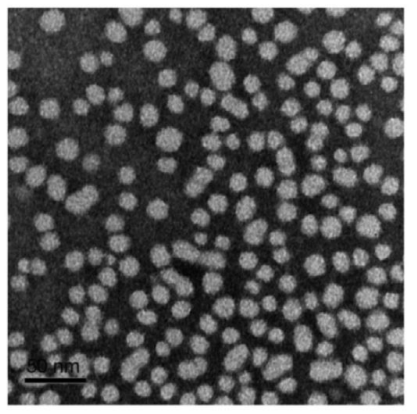

B)

Paclitaxel Release

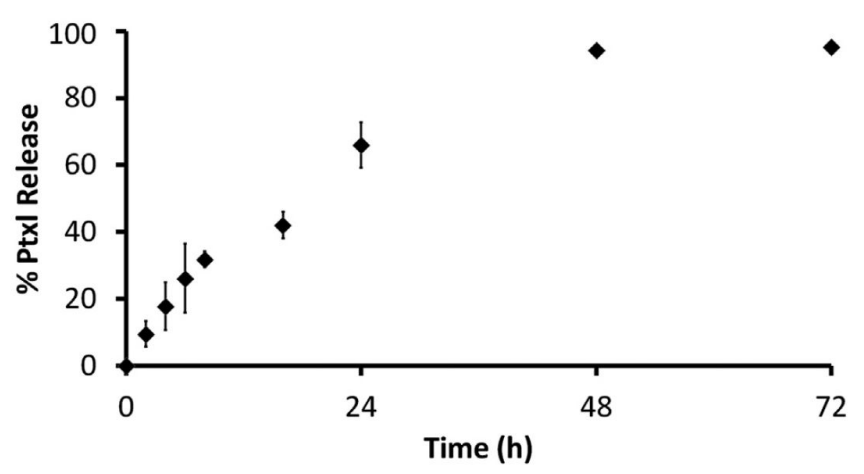

Fig. 1.

Characterization of Genexol-PM. (A) Transmission electron microscopy image of GenexolPM depicting a monodisperse population of particles with a narrow size distribution of 23.0 \pm 4.5 . (B) Drug-release curve of Genexol-PM. Genexol-PM releases paclitaxel (Ptxl) in a first-order release kinetic. Genexol-PM was incubated in phosphate buffered saline at $37^{\circ} \mathrm{C}$. 

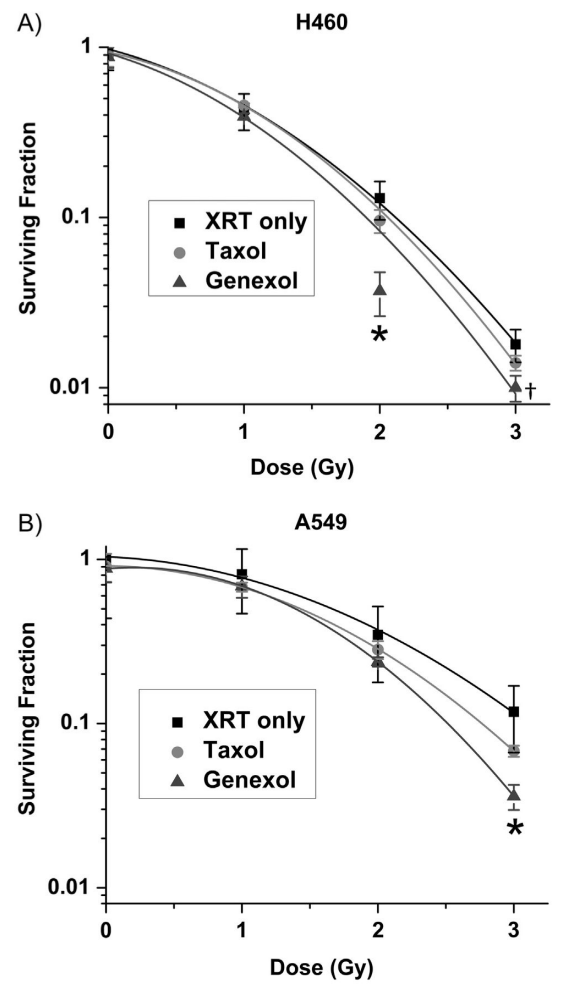

Fig. 2.

Efficacy of Genexol-PM as a radiosensitizer in non-small cell lung cancer cell lines in vitro. Radiation survival curves for (A) H460 and (B) A549 non-small cell lung cancer cells treated with saline, Taxol, or Genexol-PM. Cells were irradiated with 2 fractions of indicated doses 12 hours apart. Significant differences between Taxol and Genexol-PM at a given dose are indicated $\left(P<.01,{ }^{\dagger} P<.05\right)$. Error bars correspond to standard error of the mean ( 3 samples per time point $)$. XRT $=$ radiation therapy. 

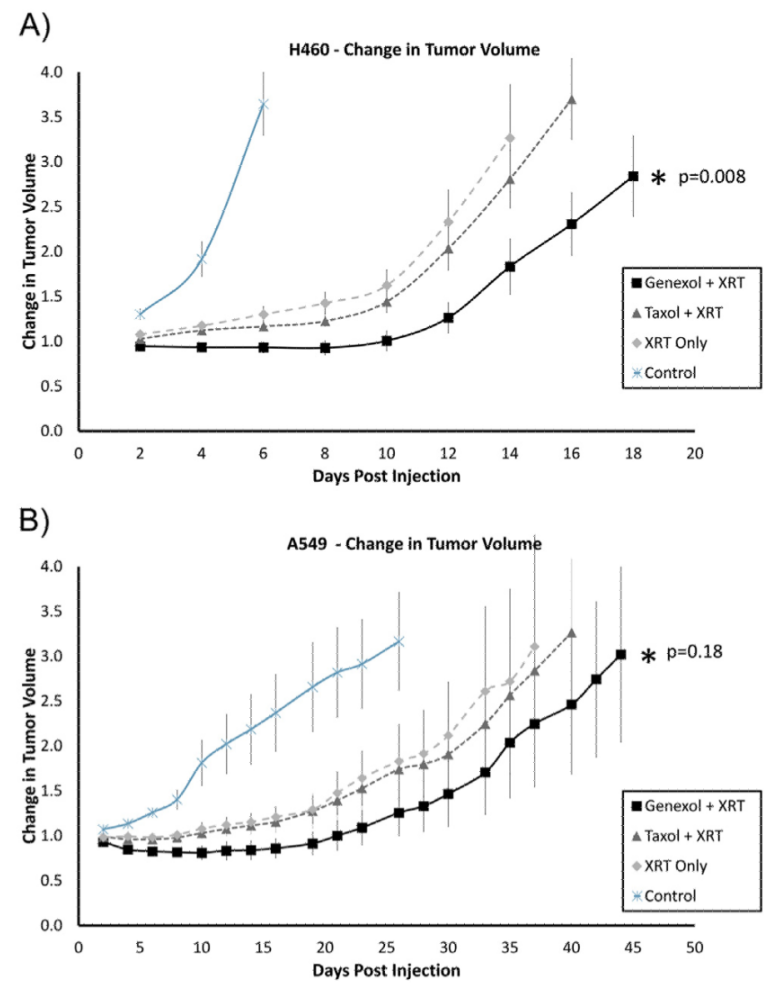

Fig. 3.

Efficacy of Genexol-PM as a radiosensitizer in chemoradiation therapy for non-small cell lung cancer cell cell lines in vivo. Mice bearing flank tumor xenografts were administered (via tail-vein intravenous injection) saline, Taxol, or Genexol-PM followed by radiation therapy (XRT). Tumors were irradiated in 5 daily fractions of 3 Gy beginning at 6 hours after chemotherapy injection. Changes in tumor volume were measured and the tumor growth delay curves for mice bearing (A) H460 and (B) A549 tumors were generated. 

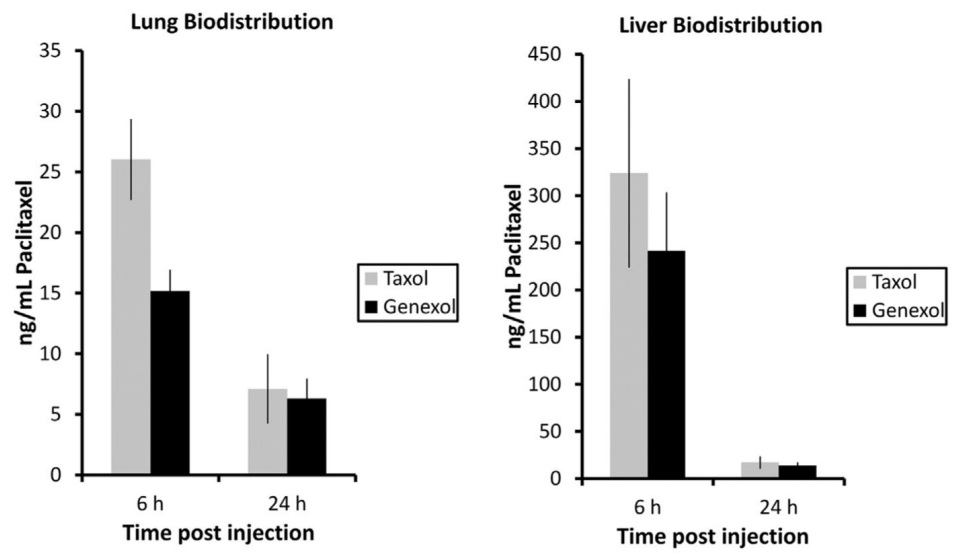

Fig. 4.

Quantification of paclitaxel dose in mouse lungs after Genexol-PM administration. Mice were administered equivalent paclitaxel doses of Taxol and Genexol-PM intravenously. Lungs were harvested at either 6 or 24 hours posttreatment, and paclitaxel dose was quantified using liquid chromatography-mass spectrometry. 